\title{
Wind Turbine Aerodynamic Load Fluctuations using Model Based Iterative Learning Control
}

\author{
Weronika N. Nowicka*, Bing Chu*, Owen R. Tutty**, and Eric Rogers*
}

\begin{abstract}
Control of aerodynamic loads in wind turbines is a critical issue in terms of keeping them economically competitive with alternative energy sources. This paper continues the investigation of the use of Iterative Learning Control (ILC) for load control in wind turbines with smart devices on rotor blades. Smart devices controlled by ILC are used to modify the blade section aerodynamics such that the fluctuations in lift due to periodic disturbances on the blades are minimized. In previous work, simple structure ILC laws were considered where the variables were chosen without the use of a model of the dynamics akin to auto-tuning design in standard control systems. This previous work demonstrated the potential of ILC in this area but, as expected, are limited in what they can deliver. This paper considers model based ILC for this application area where a Proper Orthogonal Decomposition based reduced order model of the flow is first constructed. The resulting model is used to design a norm optimal ILC scheme whose performance is evaluated in simulation.
\end{abstract}

\section{INTRODUCTION}

Wind energy is acknowledged as a cost effective and environmentally friendly source of energy. According to [1] renewables are believed to assure the energy security and mitigation of greenhouse gas emissions in future. Over the past 20 years the world total installed capacity increased from around $7 \mathrm{GW}$ to over $433 \mathrm{GW}$ with more than half of the world's wind power capacity added over past 5 years. Also the rotor diameter has increased drastically from $17 \mathrm{~m}$ in 1980 ' to $125 \mathrm{~m}$ [2]. The near future prototypes are expected to have a rotor diameter of $250 \mathrm{~m}$. In many countries, particularly in Europe, older wind turbines are being replaced with fewer, larger, taller, more efficient and more reliable machines with improved software and control mechanisms.

A developing trend in wind energy provision is to operate offshore. Reduction of cost, especially with long distance offshore operation, is a crucial issue. Possible ways of cost reduction include increasing the turbine size and therefore the energy capture, decreasing the capital cost, and lowering operation and maintenance (O\&M) costs by constructing more reliable turbines [3]. The latter can be achieved by designing effective control systems that will provide a decrease in fatigue and extreme loads on the components and lead to reduced maintenance, improved reliability and increased component lifetime. As present turbines are huge and flexible

*W. N. Nowicka, B. Chu, and E. Rogers are with the Department of Electronics and Computer Science, University of Southampton, Southampton SO17 1BJ, U.K. (e-mail: wnnlg12@ soton.ac.uk; B. Chulsoton.ac.uk; etardsoton.ac.uk).

**O. Tutty is with the Faculty of Engineering and the Environment, University of Southampton, Southampton SO17 1BJ, U.K. (e-mail ortasoton.ac.uk). structures, passive control of such structures became inefficient and more sophisticated schemes are needed. For these reasons, research on improved rotor power and load control is a very relevant topic.

Passive control is very well developed in the literature but there is also increasing interest is using Active Flow Control (AFC), for general background, see, e.g. [3], where for the application area considered one approach is based on modifying the blade section aerodynamics by incorporating smart devices, e.g., trailing-edge flaps or microtabs, which are capable to alter the local flow and therefore the lift (Fig. 1). The actuation in such systems is very fast which is a big advantage compared to traditional pitch control.

AFC requires the choice of a suitable control algorithm which is crucial for the performance of the overall system. Many approaches can be considered, ranging from a simple PID controller to advanced techniques such as Linear Quadratic Gaussian and $H_{\infty}$, Adaptive Control, Nonlinear Control, or Predictive Control, see, e.g. . [5], [6]. In [7], [8], [9] Iterative Learning Control (ILC) for load control of wind turbines with smart rotors was investigated but only modelfree ILC design was considered. This research established the feasibility of ILC in this new application for this approach to control but performance was, of course, limited by the simple structure of the control laws. Model-based controllers were recognized in this previous research as an obvious next step and provides the focus for the results reported in this paper.

The first contribution of this work is the use of Proper Orthogonal Decomposition (POD) to develop areduced order model of the flow from a Computational Fluid Dynamics (CFD) representation to provide an approximate model of the dynamics for control design. This model is then used to design a norm optimal ILC law, as one representative of possible model based design methods, to improve the aerodynamic load performance of wind turbines with smart rotors. Then the resulting controller is evaluated in simulation. The next section gives the required background.

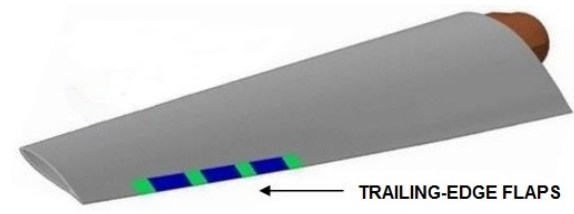

Fig. 1: Schematic of AFC devices [4]. 


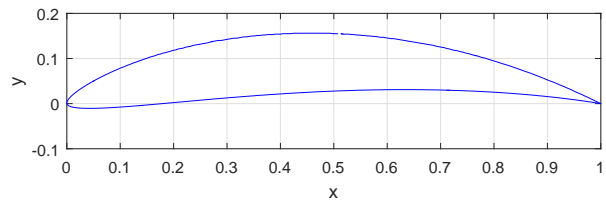

Fig. 2: Airfoil profile

\section{FLOW MODEL}

CFD uses numerical analysis to construct high quality representations of fluid flow problems. Despite the recent progress of CFD capability and development of high-speed computers, full Navier-Stokes simulation which characterizes the flow remains computationally expensive and leads to large-scale state equations. In [13], [14], as two examples, reduced order models are introduced for control design. Such models serve as low-dimensional approximations to the large-scale Navier-Stokes equations. The approximation of the system's state can be found using reduced basis functions, which can be determined using an experimental or numerical data set and the resulting model is then used for control design.

In this paper the flow past an airfoil is represented for control design using a reduced order model constructed in POD settng. The snapshot for the POD is generated using the CFD panel code from [9], [17], which simulates the flow past an airfoil (2D case). The code uses a panel method [15] with 400 vortex and 400 source panels to satisfy the boundary conditions. The problem is considered in non-dimensional form using free stream velocity $V_{\infty}$ and the chord length to normalize the time for the flow to past an airfoil and other variables, including lift and pressure.

The airfoil is generated using Kàrmàn-Trefftz [16] transform of a circle according to

$$
z=n \frac{\left(1+\frac{1}{\zeta}\right)^{n}+\left(1-\frac{1}{\zeta}\right)^{n}}{\left(1+\frac{1}{\zeta}\right)^{n}-\left(1-\frac{1}{\zeta}\right)^{n}}
$$

where $z=x+i y$ is a complex variable in the new space (airfoil profile) and $\zeta=\chi+i \eta$ is a variable in original space (circle). The parameter $n=1.9$ and the coordinates of the centre of the circle $x_{0}=-0.05$ and $y_{0}=0.2$ are used. The profile is normalized using the chord length. It is given in Fig. 2.

Remark 1: Such airfoil profile is not one of the standard types used in wind turbines, but it has a similar shape and properties (similar results were obtained using NREL S825 profile widely used for wind turbines). The conformal transformation approach was used as this simplifies greatly the calculation of the terms involved in the generation of the Reduced Order Model.

The flow past an airfoil is assumed to be inviscid (excludes extreme cases when separation is provoked) and consists of the free-stream velocity, the velocity generated by the panels and the vortices shed into the wake from the trailing edge. The motion of the vortices is found by solving the Euler equations so the position of the vortices can be tracked by solving

$$
\frac{d \mathbf{x}_{v}}{d t}=\mathbf{v}\left(\mathbf{x}_{v}, t\right)
$$

where $\mathbf{x}_{v}$ denotes the position and $\mathbf{v}\left(\mathbf{x}_{v}, t\right)$ denotes the velocity. The solution is found using second order RungeKutta method.

Finally the smart devices are modelled in a generic manner by altering the strength of the new vortex generated at each time step according to $\Gamma_{n}=-u$. This will directly change the way the flow leaves the airfoil at the trailing edge (similarly to operation of AFC devices).

It is assumed that the flow past an airfoil is periodic with the velocity equal to

$$
u_{0 x}=1+A \sin \left(\frac{2 \pi t}{T}\right)
$$

where $A$ is the amplitude of oscillation and $T$ the period of rotation. The objective of the control system is to reject the periodic disturbances in the lift and can be achieved by altering the lift on the rotor blades such that the error between the lift and the desired value for the lift is minimal

$$
e(k)=L(k)-L_{t a r}
$$

where the desired value for the lift $L_{t a r}$ can be designated by setting $A=0$ (the case without the oscillatory component).

ILC is a feedforward control approach used for systems operating in a repetitive mode and, in particular, executing the same finite task, termed a trial, over and over again. After completing each trial the system returns to its initial position ready for the next trial but the strategy can also be applied to systems where there is a stoppage between successive repetitions. The main purpose of the system is to construct the input on each trial such that as the number of trials increases the error $e_{i}(k)=y_{r e f}(k)-y_{i}(k)$, where $y_{i}(k)$ denotes the output on trial $k$ and $y_{\text {ref }}(p)$ is a supplied reference trajectory decays to zero. The design constructs the input on any trial as the sum of that used on the previous trial plus a correction term designed using the available previous trial data.

A form of ILC controller has the structure

$$
u_{i+1}(k)=u_{i}(k)+\mu e_{i}(k+\lambda)
$$

where: $u_{i}(k)$ denotes the previous input signal and $\mu>0$ and $\lambda>0$ are real scalars. This form of law is termed phase-lead due to the presence of the last term, where 'phase-lead' refers to the use (since $\lambda>0$ ) of 'advanced' error information from the previous trial, the novel feature of ILC. Such control laws, as in [7], [8], [9], can be designed without a model of the dynamics but are limited in what can be achieved. The progress in this previous research motivates consideration of model-based ILC, for which the next section describes the construction of the approximate model used in following section for norm optimal ILC design and evaluation. 


\section{Proper ORThogonal Decomposition}

POD is the method of information compression which eliminates the redundant information from the snapshot generated experimentally or numerically. As the POD functions are generated based on the data snapshot, the information in modes strictly depends on the data set and so does the ability of the modes to approximate the system's state.

In this paper we assume that the flow is periodic according to (3) therefore the total velocity field $\mathbf{u}$ can be decomposed as a sum of the steady mean flow component $\mathbf{u}_{m}$, oscillatory component and the unsteady part $\hat{\mathbf{u}}$. Also the input control part $\kappa \mathbf{u}_{c}$ is included.

$$
\mathbf{u}=\mathbf{u}_{m}(1+A \sin (\omega t))+\hat{\mathbf{u}}+\kappa \mathbf{u}_{c}
$$

where $\mathbf{u}_{m}$ is the steady mean flow, $A \sin (\omega t)$ is the oscillatory component, $\hat{\mathbf{u}}$ is the unsteady component, $\kappa$ is the sum of the control inputs from the time the circulation is turned on $\kappa=\sum_{i} u_{i}$ and $\mathbf{u}_{c}$ is the velocity field generated by an airfoil with unit circulation.

The unsteady flow $\hat{\mathbf{u}}$ can be represented by a set of POD modes $\phi_{j}(\mathbf{x})$ and their coefficients $a_{j}(t)$ [13], [14]

$$
\hat{\mathbf{u}}=\sum_{j=1}^{N} a_{j}(t) \phi_{j}(\mathbf{x})
$$

where $N$ is the number of snapshots. The coefficients $a_{j}(t)$ and modes $\phi_{j}(\mathbf{x})$ can be found by solving the eigenvalue problem $C \mathbf{a}_{j}=\lambda_{j} \mathbf{a}_{j}$ where $C_{j i}=<\hat{\mathbf{u}}_{j}, \hat{\mathbf{u}}_{i}>, \lambda_{1}, \ldots, \lambda_{N}$ are the eigenvalues sorted such that $\lambda_{1}>\lambda_{2}>\ldots>\lambda_{N}$ and $\mathbf{a}_{j}, \ldots, \mathbf{a}_{N}$ are the eigenvectors. The inner product is defined as the integral of a dot product $\langle\mathbf{a}, \mathbf{b}\rangle=\int \mathbf{a} \cdot \mathbf{b} d \mathbf{x}$.

\section{A. Derivation of Reduced Order Model}

The flow of inviscid, incompressible fluid with constant density is governed by Euler equations

$$
\left\{\begin{array}{l}
\frac{\partial \mathbf{u}}{\partial t}+\mathbf{u} \cdot \nabla \mathbf{u}=-\nabla p \\
\nabla \cdot \mathbf{u}=0
\end{array}\right.
$$

where $\mathbf{u}=(u, v)$ is the velocity vector with components in $x$ and $y$ direction and $p$ denotes the pressure.

In order to obtain a Reduced Order Model of the system the velocity field (6) with the unsteady part described by (7) is substituted back to the governing Euler equations (8). To obtain the expression for the derivatives of the coefficients $\frac{\partial}{\partial t} a_{i}(t)$ we take the inner product with respect to $\phi_{i}$ and get

$$
\frac{\partial}{\partial t} \sum_{j=1}^{N} a_{j}(t)<\phi_{i}, \phi_{j}>=\frac{\partial}{\partial t} a_{i}(t)<\phi_{i}, \phi_{i}>=\frac{\partial}{\partial t} a_{i}(t)
$$

since the modes are orthogonal such that $\left\langle\phi_{i}, \phi_{i}\right\rangle=\left\|\phi_{i}\right\|$ and are normalized such that $\left\|\phi_{i}\right\|=1$ (are orthonormal). The nonlinear terms are small and they are neglected in order to obtain the linear representation. For wake flows it is also usual to drop the pressure term $\left\langle\phi_{i},-\nabla p\right\rangle$ together with $<\phi_{i}, \mathbf{u}_{m} \cdot \nabla \mathbf{u}_{m}>$ as explained in [13], [18]. Finally the state equation has a form

$$
\begin{aligned}
\frac{\partial}{\partial t} a_{i}(t)= & -\left[\sum _ { j = 1 } ^ { N } a _ { j } ( t ) \left[( 1 + A \operatorname { s i n } ( \omega t ) ) \left(<\boldsymbol{\phi}_{i}, \boldsymbol{\phi}_{j} \cdot \nabla \mathbf{u}_{m}>\right.\right.\right. \\
& \left.\left.+<\boldsymbol{\phi}_{i}, \mathbf{u}_{m} \cdot \nabla \boldsymbol{\phi}_{j}>\right)\right]+\kappa[(1+A \sin (\omega t)) \\
& \left.\left(<\boldsymbol{\phi}_{i}, \mathbf{u}_{m} \cdot \nabla \mathbf{u}_{c}>+<\boldsymbol{\phi}_{i}, \mathbf{u}_{c} \cdot \nabla \mathbf{u}_{m}>\right)\right] \\
& +A \omega \cos (\omega t)<\boldsymbol{\phi}_{i}, \mathbf{u}_{m}>+2 A \sin (\omega t) \\
& \left.<\boldsymbol{\phi}_{i}, \mathbf{u}_{m} \cdot \nabla \mathbf{u}_{m}>-\frac{\partial}{\partial t} \kappa<\boldsymbol{\phi}_{i}, \mathbf{u}_{c} \cdot \nabla \mathbf{u}_{c}>\right]
\end{aligned}
$$

where the inner product for each term can be calculated as, e.g.

$$
\begin{aligned}
<\boldsymbol{\phi}_{i}, \mathbf{u}_{m} \cdot \nabla \mathbf{u}_{m}>= & \iint\left[\boldsymbol{\phi}_{i u}\left(\mathbf{u}_{m} \frac{\partial \mathbf{u}_{m}}{\partial x}+\mathbf{v}_{m} \frac{\partial \mathbf{u}_{m}}{\partial y}\right)\right. \\
& \left.+\phi_{i v}\left(\mathbf{u}_{m} \frac{\partial \mathbf{v}_{m}}{\partial x}+\mathbf{v}_{m} \frac{\partial \mathbf{v}_{m}}{\partial y}\right)\right] d \mathbf{x} d \mathbf{y}
\end{aligned}
$$

After completing all calculations, the result can be written in matrix form with the states $a_{i}(t)$ and input $u=\kappa$ as

$$
\frac{\partial}{\partial t} a(t)=A(t) a(t)+B_{1}(t) u(t)+B_{2} \frac{\partial}{\partial t} u(t)+O(t)
$$

The free term $O(t)$ can be removed from the state equation and included to calibrate the output instead. To eliminate the input derivative on the right-hand side of (12) the state is re-defined as $x=a-B_{2} u$ to give

$$
\frac{\partial}{\partial t} x(t)=A(t) x(t)+B(t) u(t)
$$

where $B(t)=B_{1}(t)+A(t) B_{2}$. In the following section of this paper we will use the discrete form of the equation with zero-order hold discretization of the state equation.

\section{B. Lift Estimation}

To define the output equation, the lift on the blade must be estimated. The surface of the airfoil is a streamline with the velocity tangential to the surface denoted as $u_{t}$ and the normal velocity equal to zero. Also denote the distance along the streamline by $s$. Then [15]

$$
\frac{\partial u_{t}}{\partial t}+u_{t} \frac{\partial u_{t}}{\partial s}=\frac{\partial u_{t}}{\partial t}+\frac{1}{2} \frac{\partial u_{t}^{2}}{\partial s}=-\frac{\partial p}{\partial s}
$$

Integrating it from $s_{0}=0$ to $s$ and setting $p_{0}=-\frac{1}{2} u_{t 0}^{2}$ the pressure at point $s$ can be calculated as

$$
p_{s}=-\left[\int_{0}^{s} \frac{\partial u_{t}}{\partial t} d s+\frac{1}{2} u_{t s}^{2}\right]
$$

The tangential velocity at each point on the surface can be calculated using $u$ and $v$ components of the velocity. In this research the quadratic term of the equation is linearized by re-writing the tangential velocity as

$$
u_{t}=u_{r}(\mathbf{x}, t)+\sum_{j=1}^{N} a_{j}(t) \phi_{j}(\mathbf{x})+\kappa \mathbf{u}_{c}
$$




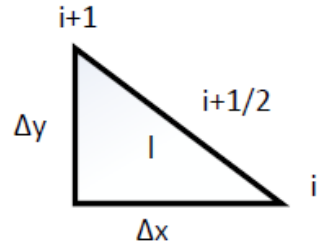

Fig. 3: Panel

where $u_{r}$ includes the mean and oscillatory parts of the flow and approximating the quadratic term as

$$
u_{t}^{2}=u_{r}^{2}(\mathbf{x}, t)+2 u_{r}(\mathbf{x}, t)\left[\sum_{j=1}^{N} a_{j}(t) \phi_{j}(\mathbf{x})+\kappa \mathbf{u}_{c}\right]
$$

In order to calculate the integral in equation (15) we divide the surface of the airfoil into $i$ panels between $i$ and $i+1$ grid points (Fig. 3). For each panel the integral is calculated as

$$
\frac{\partial}{\partial t} \int u_{t, i+\frac{1}{2}} d s=\frac{u_{t, i+\frac{1}{2}}^{k}-u_{t, i+\frac{1}{2}}^{k-1}}{\Delta t} l
$$

where $l$ is the length of the panel $l=\left[\Delta x^{2}+\Delta y^{2}\right]^{\frac{1}{2}}$ and $k$ is the time step. The lift at each panel is equal to the $y$ component of the lift and the total lift is calculated as a sum of lift on all panels. Finally the matrices for the output equation of the system can be calculated from $\mathbf{u}_{m}, \mathbf{u}_{c}$ and POD modes. The output equation will contain input and state derivative terms as shown by equation (18).

$L=C_{1}(t) a(t)+D_{1}(t) u+C_{2}\left(\frac{\partial}{\partial t} x(t)\right)+D_{2}\left(\frac{\partial}{\partial t} u(t)\right)+O_{2}(t)$

For a discrete representation those derivatives will be approximated as

$$
\frac{\partial}{\partial t} a(t)=\frac{a(k)-a(k-1)}{\Delta t}, \quad \frac{\partial}{\partial t} u(t)=\frac{u(k)-u(k-1)}{\Delta t}
$$

and substituting the state $x=a-B_{2} u$ we get

$$
L(k)=C(k) x(k)+D(k) u(k)+\hat{C} x(k-1)+\hat{D} u(k-1)+\hat{O}(k)
$$

where $C=C_{1}+C_{2}, D=D_{1}+D_{2}+C_{1} B_{2}+C_{2} B_{2}$, $\hat{C}=-C_{2}$ and $\hat{D}=-D_{2}-C_{2} B_{2}$

\section{NoRm-Optimal ILC DESIGN AND EVAluation}

Norm Optimal ILC is a model-based ILC design starting for linear dynamics from the state-space model

$$
\begin{gathered}
\mathbf{x}(k+1)=A \mathbf{x}(k)+B \mathbf{u}(k), \quad 0 \leq k \leq \alpha \\
\mathbf{y}(k)=C \mathbf{x}(k)+D \mathbf{u}(k)
\end{gathered}
$$

where $\alpha<\infty$ is the number of samples ( $\alpha$ times the sampling period gives the trial length or duration), $\mathbf{x}(k)$ is the $n \times 1$ state vector, $\mathbf{u}(k)$ is the $l \times 1$ control vector and $\mathbf{y}(k)$ is the $m \times 1$ output vector. As $\alpha$ is finite, the supervectors

$$
\mathbf{y}=\left[\begin{array}{c}
\mathbf{y}(1) \\
\mathbf{y}(2) \\
\vdots \\
\mathbf{y}(\alpha)
\end{array}\right] \mathbf{u}=\left[\begin{array}{c}
\mathbf{u}(0) \\
\mathbf{u}(1) \\
\vdots \\
\mathbf{u}(\alpha-1)
\end{array}\right]
$$

can be used to represent the dynamics in input output form as

$$
\mathbf{y}=G \mathbf{u}
$$

where

$$
G=\left[\begin{array}{cccccc}
D & 0 & 0 & 0 & \ldots & 0 \\
C B & D & 0 & 0 & \ldots & 0 \\
C A B & C B & D & 0 & \ldots & 0 \\
C A^{2} B & C A B & C B & \ldots & \ldots & 0 \\
\vdots & \vdots & \vdots & \vdots & \ddots & \vdots \\
C A^{\alpha-2} B & C A^{\alpha-3} B & \ldots & \ldots & \ldots & D
\end{array}\right]
$$

In Norm Optimal ILC a particular quadratic cost function is minimized to calculate the optimal input update. For example, choosing the cost function as

$$
J\left(\mathbf{u}_{i+1}\right)=\left\|\mathbf{e}_{i+1}\right\|_{\mathbb{Y}}^{2}+\left\|\mathbf{u}_{i+1}-\mathbf{u}_{i}\right\|_{\mathbb{U}}^{2}
$$

where $\|u\|_{\mathbb{U}}^{2}=\mathbf{u}^{T} R \mathbf{u},\|e\|_{\mathbb{Y}}^{2}=\mathbf{e}^{T} Q \mathbf{e}, R$, respectively, $Q$ is a symmetric positive definite, respectively positive semidefinite, weighting matrix. The solution (see the relevant cited papers in [10] and [11] for the details) is

$$
\mathbf{u}_{i+1}=\mathbf{u}_{i}+G^{*}\left(I+G G^{*}\right)^{-1} \mathbf{e}_{i}
$$

where $G^{*}=R^{-1} G^{T} Q$.

As the wind turbine rotor rotates the loads on the blades will contain a periodic component that arises due to effects such as wind shear or tower shadow [12]. This component will become even larger when the rotor size increases. For that reason, ILC algorithm, which uses repetitive nature of processes, can be considered as potentially beneficial for this application. As discussed in the introduction to this paper, simple model-free ILC design showed good potential in this application area [7], [8], [9] and motivates the suggestion that model-based ILC design should be considered.

In the remainder of this section, the reduced order model developed in the previous section is constructed using a data set generated in the CFD simulation and validated by comparing the reconstructed lift to the values of the lift from CFD simulation. Subsequently, the model is used to design and evaluate a norm optimal ILC scheme to reduce lift fluctuations.

\section{A. Construction and Validation of the Model}

The data set for construction of POD reduced order model was generated using the panel code [9]. An oscillatory flow of amplitude $A=0.1$ (for $x$ component only) and period $T=2.5(\omega=2.5133)$ was introduced to the flow. To obtain the modes that will perform well for different input signals the control input used to generate the snapshot was chosen as a sum of sinusoids of different frequencies

$u(t)=\mu\left(\sin \left(\frac{\omega}{3} t\right)+\sin \left(\frac{\omega}{2} t\right)+\sin (\omega t)+\sin (2 \omega t)+\sin (2.5 \omega t)\right)$

where $\mu=0.00025$.

The time step $\Delta t=0.005$ was used in the simulation but the snapshots were captured every $\Delta t_{s}=0.1$ between $t=70 \div 100$ (301 snapshots). Also the flow velocity was captured at each point of the regular grid around the airfoil, 
which was represented by a circle in computational space according to the conformal transformation (1). The regular grid around the airfoil was generated in computational space by taking $\zeta=r e^{i \theta}+\zeta_{c}$ with lines of constant radius $r$ and angle $\theta$ and then mapped into the physical space. 81 different values of the radius are used and 100 different values of the angle so the snapshot as each time consists of 8100 data points with $u$ and $v$ velocity components for each point.

For the construction of the reduced order model $N=6$ modes were used as they are capable to reconstruct over $80 \%$ of the energy in the flow (Fig. 4). The energy is calculated as

$$
E_{j}=\frac{\lambda_{j}}{\sum_{j=1}^{N} \lambda_{j}}
$$

The model was simulated for $t=0 \div 100$ operation and the comparison of lift obtained using CFD simulation and the model is shown in Fig. $5(t=50 \div 100)$. Two models were simulated: the full model of (21) and the model where the derivative terms (20) are neglected. The error of the lift at

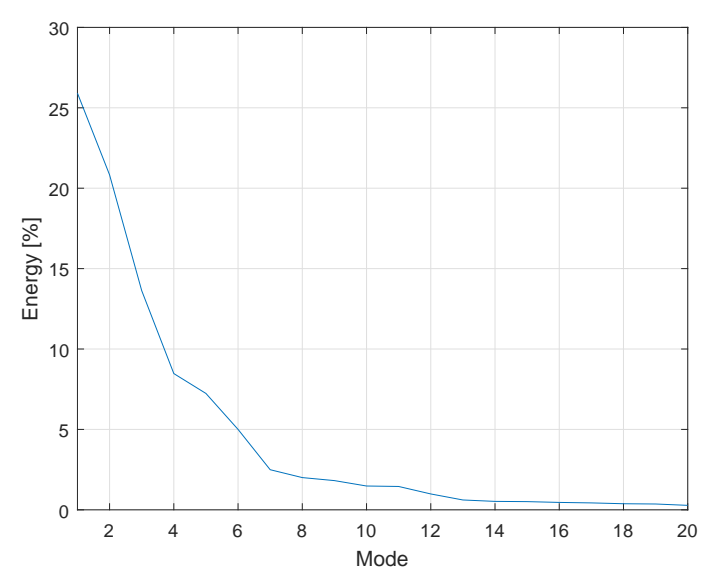

Fig. 4: Energy in the modes

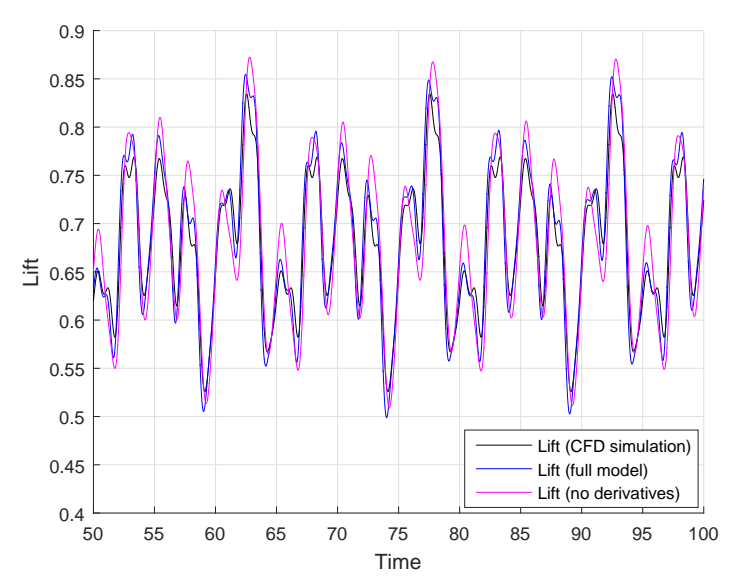

Fig. 5: Comparison of the lift obtained in CFD simulation and lift reconstructed by ROM with 6 modes each time step was calculated as

$$
\delta L[\%]=\left|\frac{L_{C F D}-L_{\text {model }}}{L_{C F D}}\right| \cdot 100 \%
$$

and the average error in the last trial was $2.1 \%$ for the full model and $2.4 \%$ while neglecting the derivatives of the input and the state.

\section{B. ILC Design}

The norm optimal ILC controller for the state-space model without derivative terms was first designed at first where in the matrix $G$ of (25) the constant matrices $A, B, C, D$ were replaced with discretized time varying matrices $A(k)$, $B_{1}(k), C_{1}(k)$ and $D_{1}(k)$ for rows/columns corresponding to input-output relation at time step $k$, i.e., the effects of the derivative terms are neglected. Diagonal weighting matrices $Q$ and $R$ were chosen and good convergence was obtained for the matrices with unit gain on the main diagonal.

Fig. 6 gives the simulation results for this design. The target value for the lift assuming no oscillatory component is $L_{t a r}=0.66$. For this design, the lift converges monotonically to the desired value for the simplified model with neglected derivative terms after two trials. The same controller applied to the model with derivative terms present does not, however, result in convergence (magenta line in the plot).

Given the conclusion above, a new NOILC controller was designed for the full model described by (13) and (21). The input-output relation of the system was calculated recursively for each time step $k$ and the new matrix $G$ including derivative terms $\hat{C}$ and $\hat{D}$ was calculated. The simulation results for this controller are given in Figs. 7 and 8.

In trial domain the mean squared error for this last design goes to zero in approximately 20 trials (Fig. 8). However, the time domain transients are not ideal in first two trials as the peaks in lift appear for the points close to beginning/end of the trials due to the change in the control input (high derivative). This effect can be minimized by average filtering the control input signal or applying the control input signal

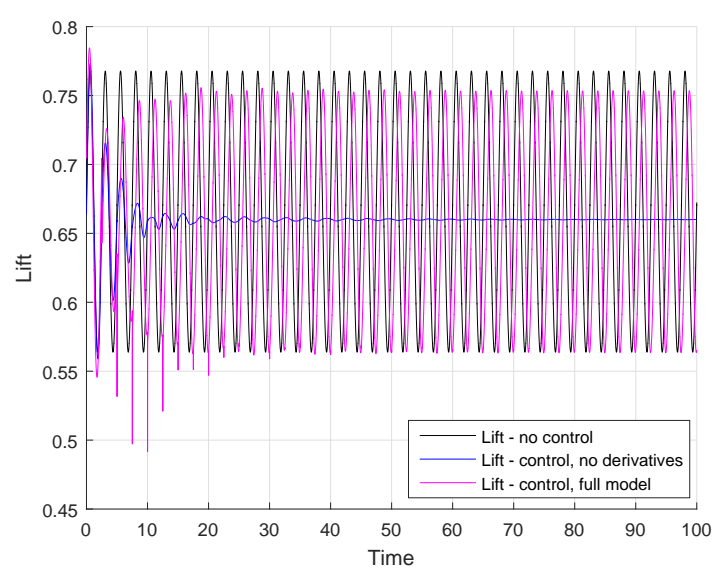

Fig. 6: Lift obtained for the system with NOILC designed neglecting derivative terms 


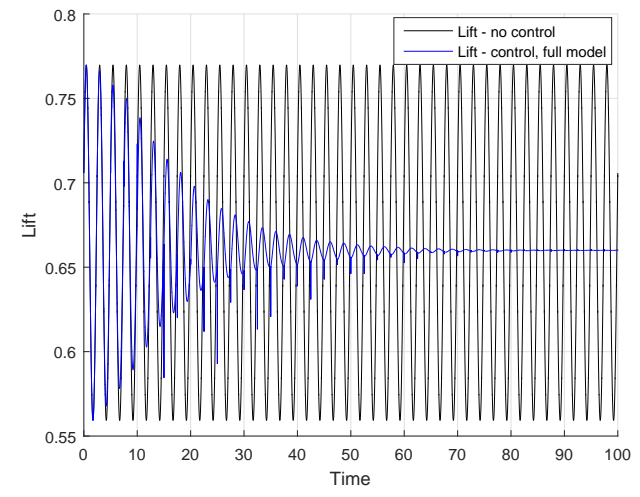

Fig. 7: Lift obtained for the system with NOILC designed for the model with derivative terms

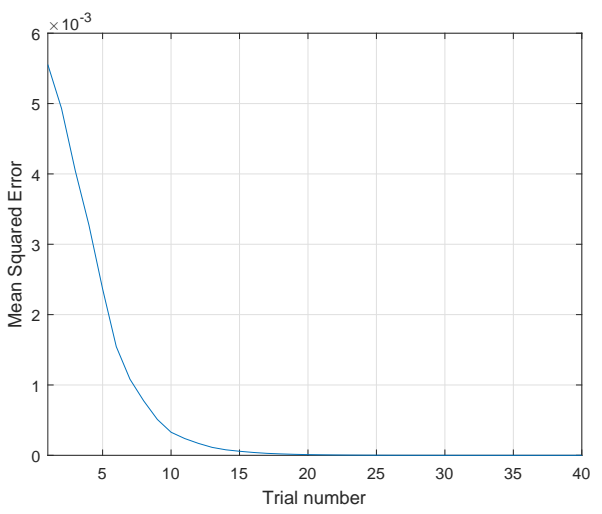

Fig. 8: Convergence of the error for the design of Fig. 7.

slowly over first few trials. To improve the robustness of the full model design the diagonal gain of the matrix $R$ was increased to three, for which the convergence is slightly slower but finally the lift reaches the target value of $L_{t a r}=$ 0.66 at around $t=80$ (Fig. 7).

\section{CONCLUSIONS}

Previous research had used simple structure model-free designs to demonstrate that ILC can be applied to aerodynamic load reduction in wind turbines by the use of smart rotors. Such controllers are limited due to their structure and an obvious next step is to consider model based design, for which this paper is the first output. The first contribution of the paper is the construction of an finite dimensional approximate model of the dynamics using a POD setting. Such a modelling approach was applied in other research to the common problem of optimal control of cylinder wake in [13], [14] but is improved in this paper to model the oscillatory, controlled flow passing the turbine blade cross section.

The model was constructed using the snapshot data obtained in the CFD simulation and validated by comparing it to the CFD simulation results. Subsequently, the model obtained was used to design norm optimal ILC schemes in two cases. Firstly by further simplifying the model through neglecting the terms including state and input derivatives and it was demonstrated that satisfactory control action was possible by this general approach. To examine the effects of the additional simplification, the design was run against the model with these terms included, resulting in much poorer performance. Completing the design for the model with these terms included showed that further progress is possible.

The results in this paper are the first on model based IC in this application area and motivate much further research. Topics for such research include other ILC design algorithms and verification against he CFD panel code.

\section{REFERENCES}

[1] REN21, "REN21's Renewables 2014-Global Status Report", 2014.

[2] L. Y. Pao, K. E. Johnson, "A Tutorial on the Dynamics and Control of Wind Turbines and Wind Farms", Proc. 2009 American Control Conference, St. Louis, MO, USA, 2009, pp. 2076-2089.

[3] S. J. Johnson, C. P. van Dam, D. E. Berg, "Active Load Control Techniques for Wind Turbines", Sandia National Laboratories, Albuquerque, NM, Rep. SAND2008-4809, Aug. 2008.

[4] D. Castaignet, N. K. Poulsen, T. Buhl, Jens J. Wedel-Heinen, "Model Predictive Control of Trailing Edge Flaps on a wind turbine blade", presented at the American Control Conf., San Francisco, CA, USA, 2011.

[5] T. K. Barlas, G. A. M. van Kuik, "Review of State of the Art in Smart Rotor Control Research for Wind Turbines", Progress in Aerospace Sciences, vol. 46, pp. 1-27, Jan. 2010.

[6] S. S. Collis, R. D. Joslin, A. Seifert, V. Theofilis, "Issues in Active Flow Control: Theory, Control, Simulation, and Experiment", Progress in Aerospace Sciences, vol. 40, pp. 237-289, May 2004.

[7] O. R. Tutty, M. Blackwell, E. Rogers, R. Sandberg, "Computational Fluid Dynamics based Iterative Learning Control of Peak Loads in Wind Turbines", presented at 51st IEEE Conference on Decision and Control, Maui, HI, USA, 2012.

[8] O. R. Tutty, M. Blackwell, E. Rogers, R. Sandberg, 'Iterative Learning Control for Improved Aerodynamic Load Performance of Wind Turbines With Smart Rotors", IEEE Trans. Control Syst. Technol., vol. 22, pp. 967-979, May. 2014.

[9] M. Blackwell, O. R. Tutty, E. Rogers, R. Sandberg, "'Iterative Learning Control Applied to a Non-Linear Vortex Panel Model for Improved Aerodynamic Load Performance of Wind Turbines with Smart Rotors", International Journal of Control, vol. 89, pp. 55-68, 2016.

[10] D. A. Bristow, M. Tharayil, A. G. Alleyne, "A Survey of Iterative Learning Control”, IEEE Control Systems Magazine, vol. 26, pp. 96114, Jul. 2006.

[11] H. S. Ahn, Y. Q. Chen, K. L. Moore, 'Iterative Learning Control: Brief Survey and Categorization", IEEE Transactions on Systems, Man, and Cybernetics, Part C (Applications and Reviews), vol. 37, pp. 10991121, Dec. 2007.

[12] I. Houtzager, J. van Wingerden, .M. Verhaegen, "Rejection of Periodic Wind Disturbances on a Smart Rotor Test Section Using Lifted Repetitive Control", IEEE Trans. Control Syst. Technol., vol. 21, pp. 347-359, Mar. 2013.

[13] M. Bergmann, L. Cordier, J. Brancher, "Optimal Rotary Control of Cylinder Wake Using Proper Orthogonal Decomposition Reduced Order Model", Physics of Fluids, vol. 17, pp. 97101-1-21, 2005.

[14] M. Bergmann, L. Cordier, J. Brancher, "Optimal Control of the Cylinder Wake in the Laminar Regime by Trust-Region Methods and POD Reduced-Order Models", Journal of Computational Physics, vol. 227, pp. 7813-7840, 2008.

[15] J. D. Anderson, "Fundamentals of Aerodynamics, Fifth Edition in SI Units", New York, NY:McGraw-Hill, 2011.

[16] R. S. Burington, "On the Use of Conformal Mapping in Shaping Wing Profiles", The American Mathematical Monthly, vol. 47, pp. 362-373, Jun. - Jul. 1940.

[17] N. R. Clarke, O. R. Tutty, "Construction and Validation of a Discrete Vortex Method for the Two-dimensional Incompressible Navier-Stokes Equations", Computer Fluids, vol. 23, pp. 751-783, Jul. 1994.

[18] B. R. Noack, K. Afanasiev, M. Morzynski, G. Tadmor, F. Thiele, "A Hierarchy of Low-Dimensional Models for the Transient and PostTransient Cylinder Wake", Journal of Fluid Mechanics, vol. 497, pp. 355-363, 2003. 